\title{
SOFT QCD MODELING OF MESON ELECTROMAGNETIC FORM FACTORS
}

\author{
PETER C. TANDY \\ Center for Nuclear Research, Department of Physics, Kent State University, Kent, \\ OH 44242, USA \\ E-mail: tandy@cnr2.kent.edu
}

\begin{abstract}
We summarize recent progress in soft QCD modeling based on the set of DysonSchwinger equations truncated to ladder-rainbow level. We pay particular attention to electromagnetic elastic and transition form factors of the pion. This covariant approach accommodates quark confinement and implements the QCD one-loop renormalization group behavior. The dressed quark propagators are compared to the most recent lattice-QCD data.
\end{abstract}

\section{Introduction}

High energy electroweak probes utilize perturbative QCD to quantify the structure of hadrons in terms of parton structure functions. Their detailed behavior samples various aspects the quark-gluon dynamics of the hadron bound state. This information is intrinsically non-perturbative and the unraveling of it from sets of structure functions will require a connection be made with non-perturbative QCD calculations and models. Although some lattice QCD studies have begun to produce moments of structure functions 1 , the opportunities are very limited at present. The most extensive hadronic models were designed to study the mass spectrum and decays and usually contain elements that limit their use for high energy lepton scattering. Examples include: non-relativistic kinematics, a lack of manifest Lorentz covariance, no quark sea, no dynamical gluons, no renormalization group behavior for change of scale, and no confinement of quarks Studies of deep inelastic scattering within the Nambu-Jona-Lasinio model 1 have helped clarify some of the issues confronting work within a quark field theory format.

Here we summarize recent progress in soft QCD modeling based on the set of Dyson-Schwinger equations [DSEs] of the theory $\mathrm{B}$; ; we pay particular attention to electromagnetic couplings to the pion. This covariant approach accommodates quark confinement and implements the QCD one-loop renormalization group behavior. Deep inelastic scattering phenomena have not been treated with the present model; a simplified related approach that retains all the essential features has recently produced excellent results for the pion valence quark distribution amplitude $\mathrm{G}$.

adel proc: submitted to World Scientific on November 10, 2018 
Meson electromagnetic form factors in impulse approximation are described by two diagrams, in one the photon couples to the quark, in the other to the antiquark. A form factor can be associated with each diagram. For example, the photon coupling to the antiquark of a flavor $a \bar{b}$ pseudoscalar meson produces 6

$$
\begin{aligned}
2 P_{\nu} F_{a \bar{b} \bar{b}}\left(Q^{2}\right)= & N_{c} \int^{\Lambda} \frac{d^{4} q}{(2 \pi)^{4}} \operatorname{Tr}\left[S^{a}(q) \Gamma_{\mathrm{ps}}^{a \bar{b}}\left(q, q_{+} ; P_{-}\right) S^{b}\left(q_{+}\right)\right. \\
& \left.\times i \Gamma_{\nu}^{b}\left(q_{+}, q_{-}\right) S^{b}\left(q_{-}\right) \bar{\Gamma}_{\mathrm{ps}}^{a \bar{b}}\left(q_{-}, q ;-P_{+}\right)\right]
\end{aligned}
$$

where $q=k+\frac{1}{2} P, q_{ \pm}=k-\frac{1}{2} P \pm \frac{1}{2} Q, P_{ \pm}=P \pm \frac{1}{2} Q$. We work in the isospin symmetry limit, so for the pion we have $F_{\pi}\left(Q^{2}\right)=F_{u \bar{u} u}\left(Q^{2}\right)$. The charged and neutral kaon form factors are given by $F_{K^{+}}=\frac{2}{3} F_{u \bar{s} u}+\frac{1}{3} F_{u \bar{s} \bar{s}}$ and $F_{K^{0}}=-\frac{1}{3} F_{d \bar{s} d}+\frac{1}{3} F_{d \bar{s} \bar{s}}$ respectively. To implement such a study, one needs a consistent QCD modeling of the photon-quark dressed vertex $\Gamma_{\nu}$, the dressed quark propagator $S(q)$, and the meson Bethe-Salpeter (BS) amplitude $\Gamma_{p s}$. The first two are related by the vector Ward-Takahashi identity[WTI]; the second two are related by the axial-vector WTI.

The DSE for the renormalized quark propagator in Euclidean space is

$$
S(p)^{-1}=i Z_{2} \not p+Z_{4} m(\mu)+Z_{1} \int^{\Lambda} \frac{d^{4} q}{(2 \pi)^{4}} g^{2} D_{\mu \nu}(k) \frac{\lambda^{a}}{2} \gamma_{\mu} S(q) \Gamma_{\nu}^{a}(q, p),
$$

where $D_{\mu \nu}(k)$ is the dressed-gluon propagator, $\Gamma_{\nu}^{a}(q ; p)$ is the dressedquark-gluon vertex, and $k=p-q$. The solution has the form $S(p)^{-1}=i p A\left(p^{2}\right)+B\left(p^{2}\right)$ and is renormalized at spacelike $\mu^{2}$ according to $A\left(\mu^{2}\right)=1$, and $B\left(\mu^{2}\right)=m(\mu)$, where $m(\mu)$ is the current quark mass. The notation $\int^{\Lambda}$ represents a translationally invariant regularization at scale $\Lambda$. The same regularization occurs consistently at all stages as indicated in Eq. (1). One takes $\Lambda \rightarrow \infty$ as the final step.

The $q \bar{q} \gamma$ vertex $\Gamma_{\mu}\left(p_{+}, p_{-}\right)$corresponding to total momentum $Q=p_{+}-p_{-}$satisfies the Bethe-Salpeter equation [BSE]

$$
\Gamma_{\mu}\left(p_{+}, p_{-}\right)=Z_{2} \gamma_{\mu}+\int^{\Lambda} \frac{d^{4} q}{(2 \pi)^{4}} K(p, q ; Q) S\left(q_{+}\right) \Gamma_{\mu}\left(q_{+}, q_{-}\right) S\left(q_{-}\right),
$$

where we use the notation $p_{+}=p+\eta Q$ and $p_{-}=p-(1-\eta) Q$ for the outgoing and incoming quark momenta respectively at the vertex. Here $\eta$, which specifies how the total momentum is shared between quark and antiquark (thus defining a relative momentum $p$ ), is arbitrary and physical observables should not depend on it. The kernel $K$ is the renormalized, amputated $q \bar{q}$ scattering kernel that is irreducible with respect to a pair of $q \bar{q}$ lines. Other 
electroweak processes $\mathrm{Q}$ require, e.g., the dressed $q \bar{q} W$ vertex which is defined in an analogous way.

When the homogeneous version of Eq. (3) has vector solutions at (discrete) timelike total momenta $Q^{2}=-m^{2}$, these are the vector meson bound states. The vector vertex $\Gamma_{\mu}\left(p_{+}, p_{-}\right)$will have simple poles at those locations. This correspondance holds for all other transformation characters (pseudoscalar, axial vector, etc), labelled by quantum numbers $J^{P C}$. The homogeneous solutions $\Gamma_{M}\left(p_{+}, p_{-}\right)$are the bound state BS amplitudes and they are normalized in the canonical way. Explicit representations of the $\Gamma_{M}\left(p_{+}, p_{-}\right)$require an expansion in a complete set of covariants constructed from gamma matrices and momenta. For example,the general representation for pseudoscalar bound states is

$$
\Gamma_{p s}\left(k_{+}, k_{-} ; P\right)=\gamma_{5}\left[i E+\not P F+\not k G+\sigma_{\mu \nu} k_{\mu} P_{\nu} H\right],
$$

where the invariant amplitudes $E, F, G$ and $H$ are Lorentz scalar functions $f\left(k^{2} ; k \cdot P ; \eta\right)$. Note that these functions depend on the momentum partitioning parameter $\eta$ because the total and relative momenta have been employed in the covariants. However, physical observables are independent of this parameter; this is verified numerically within the present approach as long as the set of employed covariants is complete 6 .

\section{Ladder-Rainbow Model}

To solve the BSE, we use a ladder truncation, with an effective quarkantiquark interaction that reduces to the perturbative running coupling at large momenta 8 . In conjunction with the rainbow truncation for the quark DSE, the ladder truncation of the BSE preserves both the vector WTI for the $q \bar{q} \gamma$ vertex and the axial-vector WTI. The latter ensures the existence of almost massless pseudoscalar mesons which are the Goldstone bosons connected with dynamical chiral symmetry breaking 6 . In combination with the impulse approximation, this ladder-rainbow truncation ensures electromagnetic current conservation 6 .

The ladder truncation of the BSE, Eq. (3), is $K(p, q ; P) \rightarrow$ $-\mathcal{G}\left(k^{2}\right) D_{\mu \nu}^{\text {free }}(k) \frac{\lambda^{i}}{2} \gamma_{\mu} \otimes \frac{\lambda^{i}}{2} \gamma_{\nu}$ where $k=p-q$, and $D_{\mu \nu}^{\text {free }}$ is the free gluon propagator in Landau gauge. The corresponding rainbow truncation of the quark DSE, Eq. (2), is given by $\Gamma_{\nu}^{i}(q, p) \rightarrow \gamma_{\nu} \lambda^{i} / 2$ together with $g^{2} D_{\mu \nu}(k) \rightarrow \mathcal{G}\left(k^{2}\right) D_{\mu \nu}^{\text {free }}(k)$. This truncation was found to be particularly suitable for the flavor octet pseudoscalar and vector mesons since the next-order contributions in a quark-gluon skeleton graph expansion, have a significant amount of cancellation between repulsive and attractive corrections 10 .

adel proc: submitted to World Scientific on November 10, 2018 
Table 1. Results

\begin{tabular}{l|cl} 
& expt. (estimates) & calc. (fitted) \\
\hline$m_{\mu=1 \mathrm{GeV}}^{u=d}$ & $5-10 \mathrm{MeV}$ & $5.5 \mathrm{MeV}$ \\
$m_{\mu=1 \mathrm{GeV}}^{s}$ & $100-300 \mathrm{MeV}$ & $125 \mathrm{MeV}$ \\
\hline$-\langle\bar{q} q\rangle_{\mu}^{0}$ & $(0.236 \mathrm{GeV})^{3}$ & $(\underline{0.241} \mathrm{GeV})^{3}$ \\
$m_{\pi}$ & $0.1385 \mathrm{GeV}$ & $\underline{0.138}$ \\
$f_{\pi}$ & $0.0924 \mathrm{GeV}$ & $\underline{0.093}$ \\
$m_{K}$ & $0.496 \mathrm{GeV}$ & $\underline{0.497}$ \\
$f_{K}$ & $0.113 \mathrm{GeV}$ & 0.109 \\
\hline$m_{\rho}$ & $0.770 \mathrm{GeV}$ & 0.742 \\
$f_{\rho}$ & $0.216 \mathrm{GeV}$ & 0.207 \\
$m_{K^{\star}}$ & $0.892 \mathrm{GeV}$ & 0.936 \\
$f_{K^{\star}}$ & $0.225 \mathrm{GeV}$ & 0.241 \\
$m_{\phi}$ & $1.020 \mathrm{GeV}$ & 1.072 \\
$f_{\phi}$ & $0.236 \mathrm{GeV}_{\phi^{2}}$ & 0.259 \\
\hline$r_{\pi}^{2}$ & $0.44 \mathrm{fm}^{2}$ & 0.45 \\
$r_{K^{+}}^{2}$ & $0.34 \mathrm{fm}^{2}$ & 0.38 \\
$r_{K^{0}}^{2}$ & $-0.054 \mathrm{fm}^{2}$ & -0.086 \\
$r_{\pi \gamma \gamma}^{2}$ & $0.42 \mathrm{fm}^{2}$ & 0.39 \\
\hline$g_{\pi \gamma \gamma}$ & 0.50 & 0.50 \\
$g_{\rho \pi \gamma}$ & 0.57 & 0.54 \\
$g_{\rho \pi \pi}$ & 6.02 & 4.85 \\
$g_{\phi K K}$ & 4.64 & 4.63 \\
$g_{K^{\star}+\pi^{+} K^{0}}$ & 4.53 & 4.6 \\
\hline & &
\end{tabular}

The model is completely specified once a form is chosen for the "effective coupling" $\mathcal{G}\left(k^{2}\right)$. We employ the Ansatz

$$
\frac{\mathcal{G}\left(k^{2}\right)}{k^{2}}=\frac{4 \pi^{2} D k^{2}}{\omega^{6}} \mathrm{e}^{-k^{2} / \omega^{2}}+\frac{4 \pi^{2} \gamma_{m} \mathcal{F}\left(k^{2}\right)}{\frac{1}{2} \ln \left[\tau+\left(1+k^{2} / \Lambda_{\mathrm{QCD}}^{2}\right)^{2}\right]},
$$

with $\gamma_{m}=12 /\left(33-2 N_{f}\right)$ and $\mathcal{F}(s)=\left(1-\exp \frac{-s}{4 m_{t}^{2}}\right) / s$. The ultraviolet behavior is chosen to be that of the QCD running coupling $\alpha\left(k^{2}\right)$; the ladderrainbow truncation then generates the correct perturbative QCD structure of the DSE-BSE system of equations. The first term implements the strong infrared enhancement in the region $0<k^{2}<1 \mathrm{GeV}^{2}$ phenomenologically required to produce a realistic value for the chiral condensate. In recent years, 
there has been some progress in understanding the interplay between ghosts and gluons in Landau-gauge QCD, indicating that ghosts could play an important role for this enhancement 6 . However, this is not yet ready for incorporation into phenomenological studies of hadron properties. We use $m_{t}=0.5 \mathrm{GeV}, \tau=\mathrm{e}^{2}-1, N_{f}=4, \Lambda_{\mathrm{QCD}}=0.234 \mathrm{GeV}$, and a renormalization scale $\mu=19 \mathrm{GeV}$ which is well into the perturbative domain 6 . The remaining parameters, $\omega=0.4 \mathrm{GeV}$ and $D=0.93 \mathrm{GeV}^{2}$ along with the quark masses, are fitted to give a good description of $\langle\bar{q} q\rangle, m_{\pi / K}$ and $f_{\pi}$. The value of $f_{K}$, the ground state vector masses and decay constants, and the other quantities in Table 11 are then produced without any free parameters 6.1 .1$. Note that the complete set of covariants of the BS amplitudes, see Eq. (畐), are needed to satisfy the axial-vector WTI $\mathrm{l}$. The pseudovector amplitudes $F$ and $G$ contribute about $30 \%$ of the masses and decay constants of the pseudoscalars; they dominate the asymptotic behavior of the pion charge form factor 15 .
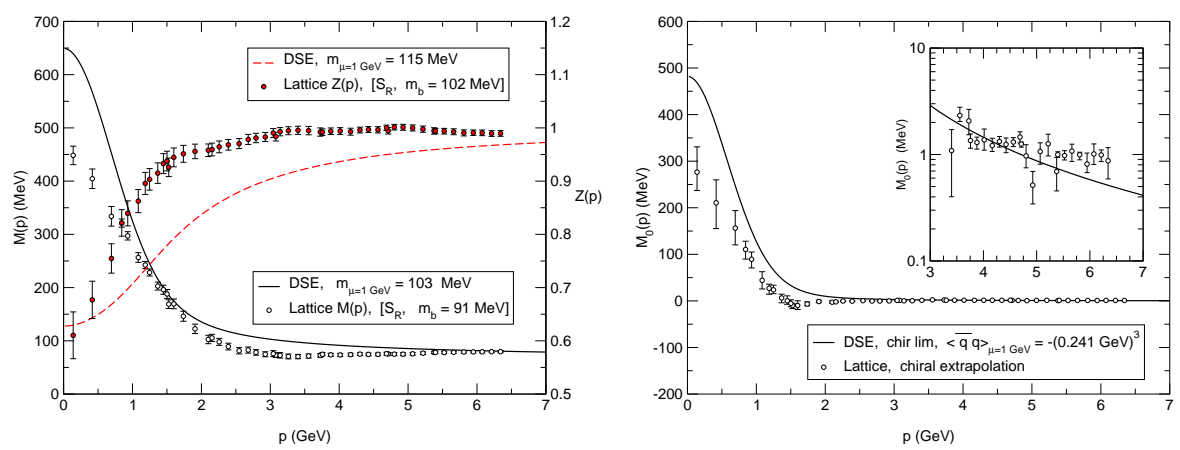

Figure 1. Left Panel: DSE solution $\mathrm{O}$ for quark propagator amplitudes compared to recent lattice data 16. Right Panel: The chiral limit DSE mass function compared to the lattice chiral extrapolation 16 .

In Fig. 11 we compare the DSE model 9 propagator amplitudes defined by $S(p)=Z\left(p^{2}\right)\left[i p+M\left(p^{2}\right)\right]^{-1}$ with the most recent results from lattice QCD 1 . In contrast to a previous comparison 17 , the data here has more lattice artifacts removed and the DSE calculations use the indicated current mass values to match the lattice mass function at $6 \mathrm{GeV}$. There is agreement in the qualitative infrared structure of the mass function particularly in the way the infrared enhancement sets in. Since the lattice simulation produces the reg- 
ulated but un-renormalized propagator, the scale of the field renormalization function $Z$ is arbitrary and only the shape is a meaningful comparison. For this reason, we have scaled the lattice data for $Z$ so that $Z(5 \mathrm{GeV})=1$. The ladder-rainbow DSE model typically produces a $Z$ that saturates much slower than does the lattice $Z$; this may signal a deficiency of the bare gluon-quark vertex. The lattice work 16 also produced a linear extrapolation to the chiral limit mass function $M_{0}(p)$ and we compare this to the DSE result with the insert emphasizing high momentum. The known one-loop renormalization group UV behavior of chiral QCD, which is preserved by this DSE model, is

$$
M_{0}\left(p^{2}\right) \simeq \frac{2 \pi^{2} \gamma_{m}}{3} \frac{-\langle\bar{q} q\rangle^{0}}{p^{2}\left(\frac{1}{2} \ln \left[\frac{p^{2}}{\Lambda_{\mathrm{QCD}}^{2}}\right]\right)^{1-\gamma_{m}}},
$$

with $\langle\bar{q} q\rangle^{0}$ being the renormalization-point-independent chiral condensate $\mathbf{6}$. Although the lattice data does not distinguish clearly between this fall-off and a flat behavior, the scale of the data is consistent with a condensate that is less than $10 \%$ higher than that of the DSE model. That is, we make a tentative assignment of $\langle\bar{q} q\rangle_{\mu=1 \mathrm{GeV}}=-(248 \mathrm{GeV})^{3}$ for this lattice data.

\section{Meson Elastic and Transition Form Factors}

Our results 6 for $F_{\pi}\left(Q^{2}\right)$ are shown in Fig. 2: the charge radii are given in Table1. Up to about $Q^{2}=3 \mathrm{GeV}^{2}$, our results for $F_{\pi}\left(Q^{2}\right)$ and $F_{K}\left(Q^{2}\right)$ can be fit-

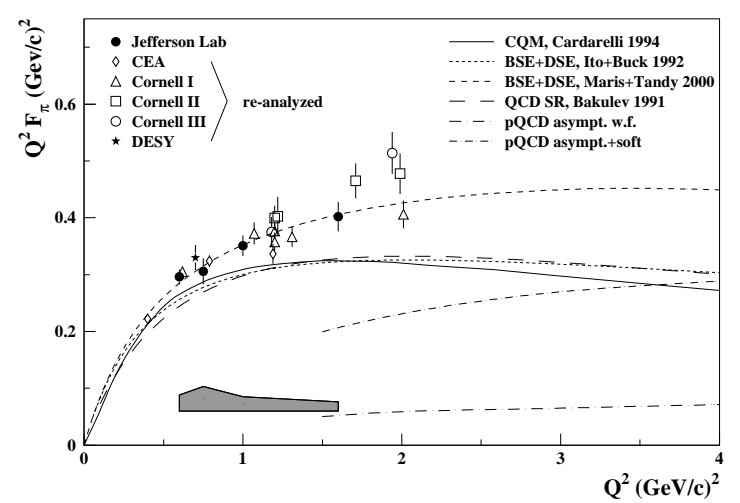

Figure 2. Our results for the pion charge form factor $\mathrm{O}$ compared to new data from JLab 18 including a re-analysis of older data. Alfo shown are various earlier model results. Figure taken from the $\mathrm{PhD}$ thesis of J. Volmer 18. 
ted quite well by a monopole 6 . Asymptotically, the behavior is $Q^{2} F\left(Q^{2}\right) \rightarrow c$ up to logarithmic corrections. However, numerical limitations prevent us from accurately determining these constants. Around $Q^{2}=3 \mathrm{GeV}^{2}$, our result for $Q^{2} F_{\pi}$ is well above the pQCD result $16 \pi f_{\pi}^{2} \alpha_{s}\left(Q^{2}\right) \sim 0.2 \mathrm{GeV}^{2}$, and clearly not yet asymptotic. The calculated time-like electromagnetic form factor displays vector meson bound state poles from which one can extract the strong coupling constants $g_{\rho \pi \pi}$ and $g_{\phi K K}$ respectively 22 . The results are given in Table 11 and are close to the experimental data and to the results from direct evaluation 20 .

The impulse approximation for the $\gamma^{\star} \pi \gamma$ vertex with $\gamma^{\star}$ momentum $Q$ is

$$
\begin{aligned}
& \Lambda_{\mu \nu}(P, Q)=i \frac{\alpha}{\pi f_{\pi}} \epsilon_{\mu \nu \alpha \beta} P_{\alpha} Q_{\beta} g_{\pi \gamma \gamma} F_{\gamma^{\star} \pi \gamma}\left(Q^{2}\right) \\
& =\frac{N_{c}}{3} \int \frac{d^{4} q}{(2 \pi)^{4}} \operatorname{Tr}\left[S(q) i \Gamma_{\nu}\left(q, q^{\prime}\right) S\left(q^{\prime}\right) i \Gamma_{\mu}\left(q^{\prime}, q^{\prime \prime}\right) S\left(q^{\prime \prime}\right) \Gamma_{\pi}\left(q^{\prime \prime}, q ; P\right)\right] .
\end{aligned}
$$

where the momenta follow from momentum conservation. In the chiral limit, the value at $Q^{2}=0$, corresponding to the decay $\pi^{0} \rightarrow \gamma \gamma$, is given by the axial anomaly and its value $g_{\pi \gamma \gamma}^{0}=\frac{1}{2}$ is a direct consequence of only gauge invariance and chiral symmetry; this value is reproduced by our calculations 15 and corresponds mell with the experimental width of $7.7 \mathrm{eV}$. In Fig. 3 (left) we show our results 13 with realistic quark masses, normalized to the experimental $g_{\pi \gamma \gamma}$. For such moderate $Q^{2}$, both the data and our DSE model are close to the
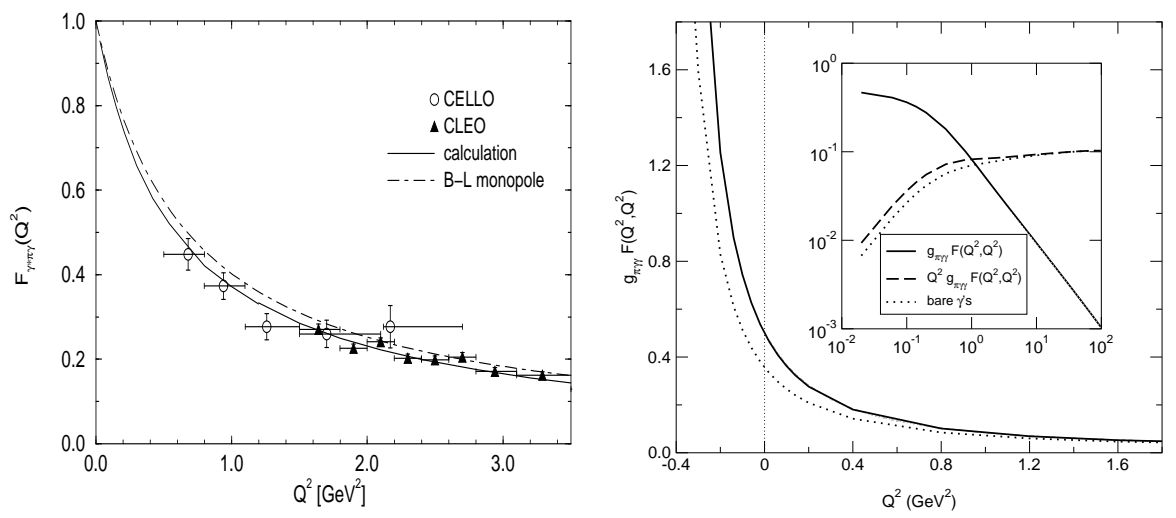

Figure 3. Left Panel: The $\gamma^{\star} \pi \gamma$ form factor 13, with data from CLEO and CELLO 21. Right Panel: The form factor for $\gamma^{\star} \pi \gamma^{\star}$ for equal virtuality with the insert showing the asymptotic behavior of $Q^{2} F\left(Q^{2}\right)$. 
monopole shape fitted to the Brodsky-Lepage asymptotic limit $22 F \sim 8 \pi^{2} f_{\pi}^{2}$ from $\mathrm{pQCD}$ in the factorized approximation. As pointed out recently 23 , it is advantageous to consider the process where pgth photons are virtual; the light-cone operator product expansion yields 22,24

$$
g_{\pi \gamma \gamma} F_{\pi \gamma \gamma}\left(Q_{1}^{2}, Q_{2}^{2}\right) \rightarrow 4 \pi^{2} f_{\pi}^{2}\left\{\frac{J(\omega)}{2\left(Q_{1}^{2}+Q_{2}^{2}\right)}+\mathcal{O}\left(\frac{\alpha_{s}}{\pi}, \frac{1}{Q^{4}}\right)\right\} .
$$

Here the asymmetry is $\omega=\left(Q_{1}^{2}-Q_{2}^{2}\right) /\left(Q_{1}^{2}+Q_{2}^{2}\right)$, and the leading coefficient

$$
J(\omega)=\frac{4}{3} \int_{0}^{1} d x \frac{\phi_{\pi}(x)}{1-\omega^{2}(2 x-1)^{2}},
$$

samples the pion bound state through the distribution amplitude $\phi_{\pi}(x)$. The normalization of the latter produces the model-independent result $J(0)=4 / 3$ for the case of equal virtuality. The model-dependent $J(1)$ for the asymmetric case has received the estimates: 2 from use of $\phi_{\pi}^{\text {asym }}(x)$ 22, 1.8 from a nonperturbative chiral quark model 23, 1.6 froma fit to CLEO data 23, and 4/3 from analysis of the impulse approximation 25, 26. A numerical confirmation of the asymptotic behavior within the present DSE model requires a level of detail in the representation of the analytic structure of dressed propagators and BS amplitudes that is not currently available. However, for equal virtuality there is no restriction, and our asymptotic result shown in Fig. 3 (right) produces $J(0)=1.2$ which is within $10 \%$ of the $4 / 3$ result of Eq. (9) from the light-cone operator product expansion.

\section{Acknowledgments}

I am grateful to the University of Adelaide's Special Research Centre for the Subatomic Structure of Matter for their hospitality and financial support. This work draws heavily upon the results of collaborations with Pieter Maris and Dennis Jarecke. I would also like to thank Craig Roberts, Tony Thomas, and Tony Williams for useful discussions. This work was partly funded by the National Science Foundation under grants Nos. PHY-0071361 and PHY9722429, and benefited from the resources of the National Energy Research Scientific Computing Center and the Ohio Supercomputer Center.

\section{References}

1. M. Gockeler, R. Horsley, E. M. Ilgenfritz, H. Perlt, P. Rakow, G. Schierholz and A. Schiller, Phys. Rev. D 53, 2317 (1996); G. Schierholz, these proceedings.

adel proc: submitted to World Scientific on November 10, 2018 
2. H. Weigel, E. Ruiz Arriola and L. Gamberg, Nucl. Phys. B 560, 383 (1999); E. Ruiz Arriola, these proceedings.

3. C. D. Roberts and S. M. Schmidt, Prog. Part. Nucl. Phys. 45, S1:1 (2000).

4. R. Alkofer and L. von Smekal, Phys. Rep. (2001), in press, hep$\mathrm{ph} / 0007355]$.

5. M. B. Hecht, C. D. Roberts and S. M. Schmidt, Phys. Rev. C 63, 025213 (2001).

6. P. Maris and P. C. Tandy, Phys. Rev. C 62, 055204 (2000)

7. C. Ji and P. Maris, in press, nucl-th/0102057.

8. P. Maris and C. D. Roberts, Phys. Rev. C 56, 3369 (1997).

9. P. Maris and P. C. Tandy, Phys. Rev. C 60, 055214 (1999).

10. A. Bender, C. D. Roberts and L. von Smekal, Phys. Lett. B 380, 7 (1996).

11. P. Maris and P. C. Tandy, Phys. Rev. C 61, 045202 (2000).

12. P. Maris and P.C. Tandy, KSUCNR-109-00, in progress, proceedings of the Confinement Research Program at the Erwin Schroedinger Institute for Mathematical Physics, Vienna, May-July, 2000.

13. P. Maris, Nucl. Phys. Proc. Suppl. 90, 127 (2000).

14. Particle Data Group, C. Caso et al., Eur. Phys. J. C 3, 1 (1998).

15. P. Maris and C. D. Roberts, Phys. Rev. C 58, 3659 (1998).

16. J. I. Skullerud and A. G. Williams, Phys. Rev. D 63, 054508 (2001); J. I. Skullerud, D. B. Leinweber and A. G. Williams, hep-lat/0102013.

17. P. Maris, nucl-th/0009064, to appear in conference proceedings; C. D. Roberts, nucl-th/0007054, to appear in conference proceedings.

18. J. Volmer, PhD thesis, Amsterdam 2000; J. Volmer et al. [The Jefferson Lab F(pi) Collaboration], Phys. Rev. Lett. 86, 1713 (2001)

19. G. R. Farrar and D. R. Jackson, Phys. Rev. Lett. 43, 246 (1979).

20. D. W. Jarecke, P. Maris and P. C. Tandy, KSUCNR-101-01, in progress, (2001).

21. H. J. Behrend et al. [CELLO Collaboration], Z. Phys. C 49, 401 (1991); J. Gronberg et al. [CLEO Collaboration], Phys. Rev. D57, 33 (1998).

22. G. P. Lepage and S. J. Brodsky, Phys. Rev. D 22, 2157 (1980).

23. I. V. Anikin, A. E. Dorokhov and L. Tomio, Phys. Lett. B 475, 361 (2000).

24. M. K. Chase, Nucl. Phys. B 167, 125 (1980).

25. D. Kekez and D. Klabucar, Phys. Lett. B 457, 359 (1999).

26. C. D. Roberts, Fizika B 8, 285 (1999); P. C. Tandy, Fizika B 8, 295 (1999); D. Klabucar and D. Kekez, Fizika B 8, 303 (1999). 